\title{
ACTIVIDAD ANTIMICROBIANA IN VITRO DEL CAMU CAMU (Myrciaria dubia) CONTRA MICROORGANISMOS ORALES: UNA REVISIÓN SISTEMÁTICA
}

\author{
Karina Pardo-Aldave 1,a, María Pareja-Vásquez ${ }^{1, b}$, Alfredo Guillén ${ }^{2, c}$, Juan Manuel Ureta-Tapia ${ }^{3, d}$
}

\section{RESUMEN}

Objetivos. Evaluar la actividad antimicrobiana de la Myrciaria dubia sobre microorganismos orales. Materiales $\boldsymbol{y}$ métodos. Se realizó una revisión sistemática de la literatura siguiendo las recomendaciones PRISMA mediante búsquedas en Pubmed, LILACS, SciELO, ProQuest, EBSCO y Google Scholar, de estudios publicados durante 2008 y 2018. Resultados. Se recopilaron 11 estudios, in vitro, todos los estudios evidenciaron actividad antimicrobiana positiva, principalmente por cada una de las partes de sus frutos, sobre grampositivos. Sin embargo, dicha actividad fue comparada con clorhexidina en solo dos estudios y en otro resultó ser mejor que un antibiótico. Se detectó un alto riesgo de sesgo en la mayoría de estudios. Los compuestos fenólicos incluidos polifenoles y acilfloroglucinoles fueron señalados como los responsables de su actividad. Conclusiones. Existe evidencia sobre la actividad antimicrobiana de M. dubia. Su estudio como antimicrobiano contra microorganismos orales es aún incipiente, pero se advierte un gran potencial debido a los fitoquímicos potentes que posee. Además, se necesita más estudios de calidad, que comparen su actividad versus antisépticos orales y sobre más microorganismos asociados a caries dental y enfermedad periodontal.

Palabras clave: Revisión; Fitoterapia; Myrtaceae; Caries dental; Microbiología; Periodontitis (fuente: DeCS BIREME).

\section{ANTIMICROBIAL ACTIVITY IN VITRO OF CAMU-CAMU (Myrciaria dubia) AGAINST ORAL MICROORGANISMS: A SYSTEMATIC REVIEW}

\begin{abstract}
Objectives. To evaluate the antimicrobial activity of Myrciaria dubia on oral microorganisms. Materials and Methods. A systematic review of the literature following PRISMA guidelines was conducted through searches of studies published between 2008 and 2018 in Pubmed, LILACS, SciELO, ProQuest, EBSCO, and Google Scholar. Results. Eleven (11) in vitro studies were gathered; all the studies showed positive antimicrobial activity on gram-positives, mainly by each of the parts of its fruits. However, such activity compared to chlorhexidine in only two studies, and, in another study, it was better than an antibiotic. A high risk of bias was detected in most studies. Phenolic compounds, including polyphenols and acylphloroglucinols, were identified as responsible for its activity. Conclusions. There is evidence of antimicrobial activity in M. dubia. Its study as an antimicrobial against oral microorganisms is still incipient, but there is great potential thanks to the potent phytochemicals it contains. Also, additional quality studies are required: comparing their activity versus oral antiseptics and on microorganisms associated with dental caries and periodontal disease.
\end{abstract}

Keywords: Review; Phytotherapy; Myrtaceae; Dental caries; Microbiology; Periodontitis (source: MeSH NLM).

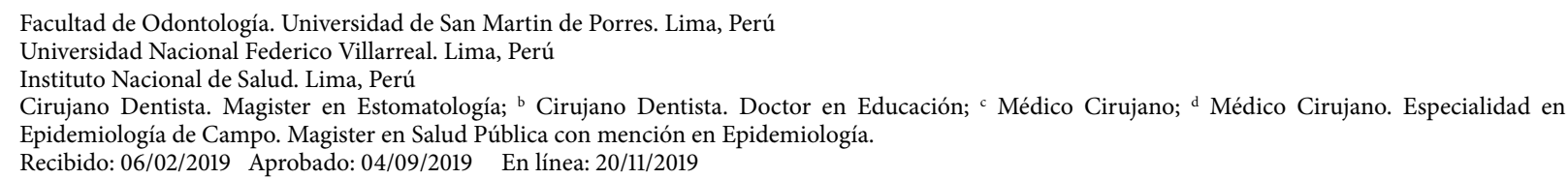

Cirujano Dentista. Magister en Estomatología; ${ }^{b}$ Cirujano Dentista. Doctor en Educación; ${ }^{c}$ Médico Cirujano; ${ }^{\mathrm{d}}$ Médico Cirujano. Especialidad en Epidemiología de Campo. Magister en Salud Pública con mención en Epidemiología.

Recibido: 06/02/2019 Aprobado: 04/09/2019 En línea: 20/11/2019

Citar como: Pardo-Aldave K, Pareja-Vásquez M, Guillen A, Ureta-Tapia JM. Actividad antimicrobiana in vitro del camu camu ( Myrciaria dubia) contra microorganismos orales: una revisión sistemática. Rev Peru Med Exp Salud Publica. 2019;36(4):573-82. doi: 10.17843/rpmesp.2019.364.4270. 


\section{INTRODUCCIÓN}

La placa dental (PD) a nivel supra y subgingival se relaciona con gingivitis, caries dental (CD) y enfermedad periodontal $(E P)$, respectivamente ${ }^{(1)}$. Sus principales agentes patógenos son el Streptococcus mutans (S. mutans) y Porphyromonas gingivalis ( $P$. gingivalis) ${ }^{(2,3)}$. Para mantener la salud de los tejidos bucales se requieren de cepillos dentales, dentíficos, hilo dental y antisépticos bucales (4-6). El gluconato de clorhexidina (CHX) es la primera elección ${ }^{(7)}$, porque conjuga los efectos antibiopelícula/antibacteriano. Pero su uso se indica por corto tiempo, debido a que presenta, tinción de dientes y trastorno temporal del gusto ${ }^{(8,9)}$. Hoy en día, la fitoterapia tiene una función potencialmente valiosa como adjunto en el manejo de la CD y EP.

La Myrciaria dubia (H.B.K) (McVaugh) "camu camu», es un arbusto frutal, nativo de la Amazonia y pertenece a la familia Myrciare. Acumula fitoquímicos que son asociados a importantes propiedades ${ }^{(10)}$. Por ejemplo, fracciones de extractos hidrometanólicos, hidroacetónicos y de extracto crudo de semilla ${ }^{(11)}$ y cáscara de $M$. dubia, evidenciaron actividad antioxidante debido a sus C-elagitaninos glucosídicos ${ }^{(11,12)}$. Asimismo, la pulpa mostró altos niveles de ácido ascórbico (AA) y gran cantidad de compuestos fenólicos ${ }^{(13)}$ y también el extracto acuoso de la cubierta de semilla de M. dubia con alto contenido fenólico ${ }^{(14)}$.

Además, un extracto metanólico de semillas de M. dubia demostró propiedad antiinflamatoria porque suprimió el edema en patas de ratones ${ }^{(15)}$ y un jugo de M. dubia al $100 \%$ redujo los marcadores inflamatorios y las IL-6 e IL-8 en fumadores con estrés oxidativo acelerado ${ }^{(16)}$. Siendo el ácido betulínico (antiinflamatorio triterpenoide) ${ }^{(15)}$, AA y fitoquímicos desconocidos ${ }^{(16)}$, los responsables de dicha propiedad, respectivamente.

Bebidas probióticas enriquecidas con compuestos fenólicos de M. dubia controlaron etapas tempranas de diabetes tipo 2 y el riesgo de hipertensión asociada ( ${ }^{(17)}$, y un extracto de la cubierta de semillas exhibió actividad antihipertensiva ${ }^{\left({ }^{14}\right)}$. Además, el extracto crudo ${ }^{(18)}$ y la pulpa ${ }^{(19)}$ de M. dubia, controlaron la obesidad ${ }^{(18,19)}$ en ratones ${ }^{(18)} y$ ratas ${ }^{(19)}$, respectivamente. También, un extracto seco de pulpa+cáscara, detuvo el crecimiento de tumores y no desarrolló mayor respuesta inflamatoria en ratas con cáncer colorrectal ${ }^{(20)}$. Finalmente, un extracto acuoso de pulpa de $M$. dubia evidenció actividad inmunoestimulante en ratas ${ }^{(21)}$.

Existen investigaciones que han comprobado su propiedad antimicrobiana, principalmente, en las áreas de Ciencias Alimentarias, Ingeniería Química y Microbiología. Pero su estudio en Odontología es incipiente. Hasta la fecha, no existe revisiones sistemáticas que estudien la actividad antimicrobiana de $M$. dubia, versus antisépticos bucales, sobre microorganismos de la cavidad oral. La CD y EP son

\section{MENSAJES CLAVE}

Motivación para realizar el estudio. La M. dubia posee una gran variedad de fitoquímicos responsables de la actividad antimicrobiana contra microorganismos orales.

Principales hallazgos. Encontramos que extractos de diferentes partes de $M$. dubia inhibieron el crecimiento y redujeron el recuento total de microrganismos orales, la bibliografía menciona que es debido a los compuestos fenólicos (incluidos polifenoles) y acilfloroglucinoles.

Implicancias. Debería ejecutarse más estudios in vitro, en un ambiente in vivo y posteriormente estudios clínicos, en vista de su potencial actividad antimicrobiana.

enfermedades crónicas complejas e inducidas por una PD patogénica ${ }^{(22)}$. Por este motivo, deben estudiarse agentes que inhiban el crecimiento de microorganismos orales, para desarrollar nuevas formulaciones preventivas/terapéuticas. El adecuado control mecánico de la acumulación de PD en dientes ha sido la piedra angular para prevenir dichas enfermedades, pero requiere de una inmensa cooperación y motivación del paciente. Por lo tanto, los agentes químicos actúan como útiles complementos para lograr un manejo efectivo de la placa dental asociada a CD y EP ${ }^{(23)}$. En consecuencia, el objetivo del presente estudio tuvo como objetivo evaluar la actividad antimicrobiana in vitro de $M$. dubia contra microorganismos orales.

\section{MATERIALES Y MÉTODOS}

\section{PREGUNTA DE INVESTIGACIÓN}

La presente revisión sistemática se diseñó acorde al reporte PRISMA (Preferred Reporting Items for Systematic Reviews and Meta-Analyses) (24) para responder la pregunta, formulada según el formato PICO ¿La Myrciaria dubia (Intervención) ejerce una verdadera actividad antimicrobiana (Evento/Outcome), comparada con controles positivo y/o negativo (Comparación), sobre microorganismos orales (Problema) en estudios experimentales in vitro (Diseño de estudio/Study design)?

\section{CRITERIOS DE SELECCIÓN}

Tipos de estudios. Estudios experimentales in vitro publicados en los últimos diez años ( 1 enero de 2008 al 31 diciembre de 2018) porque son los estudios más recientes, rigurosos y contienen los datos más relevantes. Estudios, que investigaron la actividad antimicrobiana de $M$. dubia versus controles, contra microorganismos orales, sin restricción de idioma y ámbito geográfico.

Tipos de participantes. Microorganismos orales involucrados en la etiología y progresión de CD y EP (Streptococcus 
mutans, Streptococcus sanguinis, Streptococcus sobrinus, Candida albicans, Porphyromonas gingivalis, Fusobacterium nucleatum, Aggregatibacter actinomycetemcomitans, Tannarella forsythia, Treponema denticola o Staphylococcus aureus). Utilizando cultivos de cepas o muestras de saliva o placa dental de humanos.

Tipos de intervención. Extractos (alcohólicos, hidroalcohólicos y acuosos), fracciones de extractos, fitoquímicos aislados, zumos y/o antisépticos (soluciones) provenientes del fruto o tejidos/órganos de $M$. dubia versus controles positivos (clorhexidina, cloranfenicol, antisépticos orales comerciales, antibióticos y antifúngicos) y controles negativos (soluciones placebo).

Tipos de mediciones del evento. Evento primario: actividad antimicrobiana, determinada mediante métodos de susceptibilidad antimicrobiana, método de difusión en agar (disco o pocillo) y microdilución en caldo. Los resultados fueron cuantificados mediante la medición de la zona de inhibición del crecimiento microbiano $(\mathrm{mm})$ y concentración inhibitoria mínima (CIM), respectivamente. Evento secundario: Identificación de fitoquímicos responsables de la actividad antimicrobiana de M. dubia.

\section{CRITERIOS DE EXCLUSIÓN}

Tipos de estudios. Revisiones de literatura, reporte de caso, proyectos/protocolos, comunicaciones cortas, opiniones personales, cartas, posters, resúmenes de conferencias y estudios in vivo.

Tipos de intervención. Productos farmacéuticos (como tabletas, cápsulas) oextractos que contengan combinaciones de $M$. dubia con otros productos farmacéuticos u otras plantas medicinales.

\section{MÉTODOS DE BÚSQUEDA PARA LA IDENTIFICACIÓN DE ESTUDIOS}

Información de las bases de datos: La búsqueda de los artículos científicos se realizó en bases de datos electrónicas, por cada una de las intervenciones predefinidas, entre el 11 de julio y 15 de setiembre de 2018. No se aplicó limites en el idioma y artículos extranjeros fueron traducidos.

Las bases de datos y buscadores utilizados fueron PubMed; ProQuest; SciELO (Scientific Electronic Library Online); LILACS (Latin American and Caribbean Health Sciences Literature); EBSCOhost, Google Scholar.

Estrategias de búsqueda. Se elaboró un modelo de búsqueda para PubMed, usando términos controlados del $\mathrm{MeSH}$ (Medical Subject Headings) y términos libres. Para las demás bases de datos se adaptó este modelo y usaron términos libres basados en los términos controlados del MeSH o DECS (Descriptores en Ciencias de la Salud) y/o una combinación del vocabulario controlado con términos libres, teniendo en cuenta las diferencias en vocabulario controlado y reglas de sintaxis (Anexo 1).

\section{EXTRACCIÓN Y ANÁLISIS DE LOS DATOS}

Extracción de datos. En cada artículo obtenido por las estrategias de búsqueda, dos autores (KPA y MPV) aplicaron los criterios de selección/exclusión al resumen, título o a ambos y los revisaron de manera simultánea e independiente; sólo con los estudios en que hubo duda se leyó el texto completo. Todos los estudios potencialmente relevantes fueron buscados en texto completo. Con aquellos que no cumplieron los criterios de selección fueron registrados como artículos excluidos de la revisión y se anotó la razón de su exclusión. Cualquier discrepancia fue resuelta a través de consenso con un tercer y cuarto revisor (AG y JMUT).

Los eventos primario y secundario fueron la actividad antimicrobiana de $M$. dubia y la identificación de fitoquímicos responsables de dicha actividad, respectivamente. Adicionalmente, se extrajo información sobre aspectos como el nombre del autor principal, año de publicación, país de origen del estudio, formulación farmacológica, parte/tejido/órgano y procedencia de la M. dubia, grupo control, microorganismos orales estudiados y método para evaluar la actividad antimicrobiana. En la Figura 1 se muestra el proceso de selección de los estudios de acuerdo a la declaración PRISMA. También se extrajo información acerca de la actividad antimicrobiana de $M$. dubia y controles.

Artículos relevantes identificados después de remover los repetidos

$$
\mathrm{n}=2679
$$

\begin{tabular}{|c|c|}
\hline $\begin{array}{c}\text { Artículos } \\
\text { potencialmente } \\
\text { relevantes en texto } \\
\text { completo para } \\
\text { análisis } \\
\mathrm{n}=92\end{array}$ & $\begin{array}{c}\text { Artículos excluidos por } \\
\text { título/resumen } \\
\mathrm{n}=2587\end{array}$ \\
\cline { 2 - 3 } & $\rightarrow \begin{array}{c}\text { Artículos excluidos } \\
\text { No evaluaron } \\
\text { bacterias orales }=25 \\
\text { No usaron } \\
M . \text { dubia }=49 \\
\text { Diseño experimental } \\
\text { inapropiado }=3 \\
\text { Grupo control } \\
\text { inapropiado } \mathrm{n}=1 \\
\text { Revisión }=3\end{array}$ \\
\hline $\begin{array}{c}\text { Artículos incluidos y } \\
\text { evaluados } \\
\mathrm{n}=11\end{array}$ & \\
\hline
\end{tabular}

Figura 1. Flujograma de los estudios incluidos 
Evaluación del riesgo de sesgo de los estudios incluidos. Dos investigadores (KPA y AG) evaluaron, de forma independiente, el riesgo de sesgo de los estudios seleccionados, siguiendo los criterios propuestos por el Instituto Joanne Briggs (Institute Joanna Briggs, 2014) ${ }^{(25)}$. Un tercer revisor estuvo involucrado en caso de discrepancia (JMUT). La escala tiene diez preguntas, pero no se consideraron dos, debido a que no cumplieron con el análisis de sesgo para estudios in vitro. Por lo tanto, se evaluaron las siguientes preguntas:

¿La asignación al grupo de tratamiento fue verdaderamente aleatoria? ¿La asignación a los grupos de tratamiento se ocultó del asignador? ¿Los que evaluaban los resultados estaban cegados a la asignación al tratamiento? ¿Los grupos de control y tratamiento eran comparables al inicio? ¿Los grupos fueron tratados de manera idéntica por las intervenciones mencionadas? ¿Se midió el evento de la misma manera para todos los grupos? ¿El evento se midió de manera confiable? ¿Se utilizó el análisis estadístico apropiado?

Se respondió a cada pregunta con un «sí», cuando hubo suficiente información disponible, siendo equivalente a un bajo riesgo de sesgo. En caso de información inexistente, se dio la respuesta «no», que equivale a un alto riesgo de sesgo. La respuesta «no claro» se atribuyó cuando no se pudo clasificar el riesgo de sesgo como alto o bajo (Figura 2).

Buscando heterogeneidad. Se determinó en función a la intervención (características de la M. dubia y tipos de controles) y el evento (metodología) estudiados en cada uno de los artículos seleccionados. De esta manera, no se pudo llevar a cabo un metanálisis porque los artículos mostraron una alta heterogeneidad, lo que imposibilitó hacer comparaciones similares para evaluar el evento principal.

\section{RESULTADOS}

Se recuperaron 4908 títulos/resúmenes, de los cuales se excluyeron 2229, por estar duplicados. En función a los criterios de selección, se evaluaron 92 títulos completos y 11 fueron seleccionados ${ }^{(26-36)}$. Las razones por la que se excluyeron estudios son mencionadas en la Figura 1.

Las características principales por los que se seleccionó los estudios y sus resultados más relevantes se presentan en la Tabla 1.

Se utilizaron diferentes partes de M. dubia (en orden correlativo, cáscara ${ }^{(26-30)}$, pulpa ${ }^{(31-33)}$, semilla ${ }^{(26,29,33)}$, hojas ${ }^{(34,35)}$, corteza ${ }^{(34)}$, una combinación de cáscara + semilla ${ }^{(36)}$ y se aislaron cuatro fitoquímicos ${ }^{(28,29)}$. Todos los estudios fueron in vitro ${ }^{(26-36)}$ y uno de ellos incluyó un in vivo ${ }^{(35)}$. Se aplicaron dos métodos para evaluar la

\begin{tabular}{|c|c|c|c|c|c|c|c|c|}
\hline Myoda T, 2010 & $?$ & $?$ & $?$ & + & + & + & + & \\
\hline Fujita A, 2013 & $?$ & $?$ & $?$ & + & + & + & + & $?$ \\
\hline Mori T, 2013 & $?$ & $?$ & $?$ & + & + & + & + & - \\
\hline Castillo-Carranza CN, 2013 & $?$ & $?$ & $?$ & + & + & + & + & $?$ \\
\hline Silva de Azevedo JC, 2014 & $?$ & $?$ & $?$ & + & + & + & + & $?$ \\
\hline Moromi-Nakata H, 2014 & $?$ & $?$ & $?$ & + & + & + & + & \\
\hline Fujita A, 2015 & $?$ & $?$ & $?$ & + & + & + & + & ? \\
\hline Kaneshima T, 2015 & $?$ & $?$ & $?$ & + & + & + & + & \\
\hline Camere-Colarossi RV, 2016 & $?$ & $?$ & $?$ & + & + & + & + & $?$ \\
\hline Saldarriaga Mostacero, 2017 & $?$ & $?$ & $?$ & + & + & + & + & + \\
\hline Kaneshima T, 2017 & $?$ & $?$ & $?$ & + & + & + & + & \\
\hline
\end{tabular}

P1: ¿La asignación al grupo de tratamiento fue verdaderamente aleatoria?

P2: ¿La asignación a los grupos de tratamiento se ocultó del asignador?

P3: ¿Los que evaluaban los resultados estaban cegados a la asignación al tratamiento?

P4: ¿Los grupos de control y tratamiento eran comparables al inicio?

P5: ¿Los grupos fueron tratados de manera idéntica por las intervenciones mencionadas?

P6: ¿Se midió el evento de la misma manera para todos los grupos?

P7: ¿El evento se midió de manera confiable?

P8: ¿Se utilizó el análisis estadístico apropiado?

\begin{tabular}{|c|c|c|}
\hline «Si» & I Hubo suficiente información & I Bajo riesgo de sesgo \\
\hline «No» & I Existe bastante información & | Alto riesgo de sesgo \\
\hline «No claro» & $\begin{array}{l}\text { No se pudo clasificar el riesgo } \\
\text { de sesgo como alto o bajo }\end{array}$ & \\
\hline & $\begin{array}{l}\text { No se ejecutaron análisis } \\
\text { estadístico }\end{array}$ & \\
\hline
\end{tabular}

Figura 2. Riesgo de sesgo de los estudios incluidos

actividad antimicrobiana contra microorganismos orales (evento primario) por microdilución en caldo ${ }^{(27-34,36)}$ y difusión en agar (disco ${ }^{(26,27,30,34,35)}$ o pocillo ${ }^{(31-33,36)}$ ), cuyos resultados cuantificaron la concentración inhibitoria mínima (CIM) ${ }^{(27-34,36)}$ y la zona de inhibición del crecimiento microbiano ${ }^{(26,27,30-36)}$, respectivamente y el método menos usado fue la unidad formadora de colonias (UFC) ${ }^{(30)}$. Se identificaron a compuestos fenólicos y lipofílicos, polifenoles y acilfloroglucinoles, como los fitoquímicos responsables de la actividad antimicrobiana de $M$. dubia (evento secundario).

La M. dubia provino principalmente de Perú ${ }^{(26-30,33-35)}$ y Brasil $(31,32,36)$. Mientras que, sus formulaciones fitofarmacológicas más utilizadas fueron los extractos alcohólicos (etanol ${ }^{(27,30)}$, hexano ${ }^{(28,29)}$, y metanol $\left.{ }^{(33)}\right)$, hidroalcohólicos ${ }^{(26,31,34,35)}$ y acuosos ${ }^{(32,36)}$. Cuyos efectos se estudiaron contra el S. aureus ${ }^{(26-29,31,32,34,36)}$, S. mutans ${ }^{(28-30,33,35)}$, C. albicans ${ }^{(27-29)}$, $P$. gingivalis ${ }^{(35)}$ y $S$. sanguinis ${ }^{(33)}$. También, nos muestra que la aplicación de un colutorio de $M$. dubia en sujetos voluntarios redujo el recuento de la flora microbiana hasta diez minutos. Además, es importante mencionar que los estudios ejecutados mostraron resultados positivos, respecto a la seguridad de $M$. dubia, ya que no se mencionaron reacciones adversas visibles, en el uso de un colutorio de hojas, en humanos ${ }^{(35)}$ y extractos metanólicos de semilla y pulpa no fueron citotóxicos en altas concentraciones ${ }^{(33)}$. 
Tabla 1. Características y resultados principales de los estudios in vitro incluidos

\begin{tabular}{|c|c|c|c|c|c|c|c|}
\hline $\begin{array}{l}\text { Primer autor/ } \\
\text { año/ país/ } \\
\text { Formato }\end{array}$ & $\begin{array}{l}\text { Formulación/ } \\
\text { fracción (Fr)/ parte/ } \\
\text { Procedencia de } M \text {. } \\
\text { dubia }\end{array}$ & $\begin{array}{l}\text { Grupo control } \\
\text { (Co) }\end{array}$ & $\begin{array}{l}\text { Evaluación de } \\
\text { la actividad } \\
\text { antimicrobiana }\end{array}$ & Microorganismos & $\begin{array}{l}\text { Resultados principales de } \\
\text { M. dubia y controles/ Tamaño de } \\
\text { muestra (n) }\end{array}$ & $\begin{array}{l}\text { ¿Diferencia } \\
\text { significativa } \\
\text { entre } M . \text { dubia } \\
\text { y control? } \\
\text { (valor de p) }\end{array}$ & Referencia \\
\hline \multirow[b]{2}{*}{$\begin{array}{l}\text { Myoda T, } \\
\text { et all 2010/ } \\
\text { Japón } \\
\text { (Artículo) }\end{array}$} & \multirow{2}{*}{$\begin{array}{c}\text { Extracto } \\
\text { hidroacetónico }(\mathrm{EH}) \\
\text { de semilla (Perú) } \\
\text { EH de cáscara } \\
\text { (Perú) }\end{array}$} & \multirow[b]{2}{*}{ Kanamicina } & \multirow[b]{2}{*}{$\begin{array}{l}\text { Difusión en agar } \\
\text { con disco }\end{array}$} & \multirow[b]{2}{*}{$\begin{array}{l}\text { S. aureus ATCC } \\
11522\end{array}$} & $\begin{array}{l}\text { M. dubia de } 5,0 \mathrm{mg} / \mathrm{mL}: 2,7 \mathrm{~mm} ; M \text {. } \\
\text { dubia } \mathrm{Fr}-100 \% \text { de } 5,0 \text { y } 1,0 \mathrm{mg} / \mathrm{mL} \text { : } \\
4,7 \text { y } 3 \mathrm{~mm} \text {, respectivamente. }(\mathrm{n}=3)\end{array}$ & \multirow[b]{2}{*}{ No } & \multirow[b]{2}{*}{26} \\
\hline & & & & & $\begin{array}{c}\text { M. dubia de } 5,0 \mathrm{mg} / \mathrm{mL}: \\
3,1 \mathrm{~mm} ; \text { M. dubia } \mathrm{Fr}-100 \% \text { a } 5,0 \text { y } 1,0 \\
\mathrm{mg} / \mathrm{mL}: 3,8 \text { y } 2,0 \mathrm{~mm} \text {, respectivamente. } \\
\text { Control }(\mathrm{Co}): \\
3,3 \mathrm{~mm} .(\mathrm{n}=3)\end{array}$ & & \\
\hline $\begin{array}{l}\text { Fujita A, et all } \\
2013 / \text { Brasil } \\
\text { (Artículo) }\end{array}$ & $\begin{array}{c}\text { Extracto } \\
\text { hidrometanólico } \\
\text { (EHM) de pulpa } \\
\text { liofilizada (Brasil) } \\
\text { EHM de pulpa } \\
\text { secada por lecho } \\
\text { de fuente fluidizado } \\
\text { (Brasil) }\end{array}$ & Ampicilina & $\begin{array}{l}\text { Difusión en } \\
\text { agar con pocillo } \\
\text { y Método de } \\
\text { microdilución }\end{array}$ & $\begin{array}{l}\text { S. aureus ATCC } \\
29213\end{array}$ & $\begin{array}{c}\text { M. dubia } 60 \text { Co y } 0 \% \text { maltodextrina: } \\
19 \pm 1 \mathrm{~mm} \text { y CIM: } 0,08 \mathrm{mg} / \mathrm{mL} \text {. CIM del } \\
\text { Co: } 0,26 \mathrm{mg} / \mathrm{mL}(\mathrm{n}=3)\end{array}$ & $\begin{array}{l}\text { No } \\
\text { determinado }\end{array}$ & 31 \\
\hline $\begin{array}{l}\text { Mori T, et al./ } \\
\text { 2013/ Perú } \\
\text { (Artículo) }\end{array}$ & $\begin{array}{c}\text { Extracto } \\
\text { hidroetanólico } \\
\text { liofilizado (EHEL) de } \\
\text { hojas (Perú) } \\
\text { EHEL de corteza } \\
\text { (Perú) }\end{array}$ & Ampicilina & $\begin{array}{l}\text { Difusión en agar } \\
\text { con discos y } \\
\text { ensayo en tubos y } \\
\text { dilución }\end{array}$ & $\begin{array}{l}\text { S. aureus ATCC } \\
6538 \mathrm{P}\end{array}$ & $\begin{array}{c}\text { M. dubia de } 500,700 \text { y } \\
800 \mathrm{mg} / \mathrm{mL} \text { : mostraron actividad } \\
\text { antimicrobiana y } 15 \mathrm{~mm} \\
\text { (mayor halo) y CIM (sobre una } \\
\text { suspensión de } 10^{6} \mathrm{UFC} / \mathrm{mL} \text { ): } \\
6,38 \mathrm{mg} / \mathrm{mL} \text {. Co: no menciona. } \\
\text { ( } \mathrm{n}=\text { no menciona) }\end{array}$ & $\begin{array}{l}\text { No } \\
\text { determinado }\end{array}$ & 34 \\
\hline $\begin{array}{l}\text { Castillo- } \\
\text { Carranza } \\
\text { CN, et al./ } \\
\text { 2013/ Perú } \\
\text { (Tesis) }\end{array}$ & $\begin{array}{l}\text { Extracto etanólico de } \\
\text { cáscara (Perú) }\end{array}$ & $\begin{array}{l}\text { Vancomicina y } \\
\text { fluconazol }\end{array}$ & $\begin{array}{l}\text { Difusión en } \\
\text { agar con disco } \\
\text { y Método de } \\
\text { dilución en tubos } \\
\text { abreviado }\end{array}$ & $\begin{array}{l}\text { S. aureus ATCC } \\
10231 \\
\text { C. albicans ATCC } \\
25923\end{array}$ & $\begin{array}{l}\text { M. dubia Fr-100\%: } 23 \pm 69 \text { mm y CIM } \\
\text { (M. dubia Fr-75\%): 0,00 UFC. }(n=10) \\
\text { M. dubia Fr-100\%: } 21 \pm 91 \text { mm y CIM } \\
\text { (M. dubia Fr-100\%): 0,00 UFC. Co: no } \\
\text { menciona ninguno. }(n=10)\end{array}$ & $\begin{array}{c}\text { No } \\
\text { determinado }\end{array}$ & 27 \\
\hline $\begin{array}{l}\text { De Azevêdo } \\
\text { JCS, } \\
\text { et al./ 2014/ } \\
\text { Brasil } \\
\text { (Artículo) }\end{array}$ & $\begin{array}{l}\text { Extracto acuoso de } \\
\text { cáscara+semilla } \\
\text { (EACS), frescas } \\
\text { (Brasil) } \\
\text { EACS secadas por } \\
\text { liofilización (Brasil) }\end{array}$ & Ampicilina & $\begin{array}{l}\text { Difusion en } \\
\text { agar con pocillo } \\
\text { y Método de } \\
\text { microdilución }\end{array}$ & $\begin{array}{l}\text { S. aureus ATCC } \\
29213\end{array}$ & $\begin{array}{c}\text { M. dubia Fr rica en polifenoles } \\
\text { (obtenidad por método } 2) \text { : } \\
16 \pm 1 \mathrm{~mm} \text { y CIM: } 2,5 \mathrm{mg} / \mathrm{mL}(\mathrm{n}=3) \\
\text { M. dubia Fr rica en polifenoles (método } \\
\text { 2): } 15 \pm 1 \mathrm{~mm} \text { y CIM: } \\
2,5 \mathrm{mg} / \mathrm{mL} \text {, respectivamente. CIM del } \\
\text { Co: } 0,25 \mathrm{mg} / \mathrm{mL} \text {. }(\mathrm{n}=3)\end{array}$ & $\begin{array}{l}\text { No } \\
\text { determinado }\end{array}$ & 36 \\
\hline \multirow[t]{2}{*}{$\begin{array}{l}\text { Fujita A, } \\
\text { et al./ 2015/ } \\
\text { Brasil } \\
\text { (Artículo) }\end{array}$} & $\begin{array}{c}\text { Extracto acuoso } \\
\text { de pulpa liofilizada } \\
((\text { EAPL) de } \\
\text { Amazonas } \\
\text { EAPL de Sao Paulo } \\
\text { Extracto acuoso de } \\
\text { pulpa secada por } \\
\text { aspersion (EAPA) de } \\
\text { Amazonas }\end{array}$ & \multirow[t]{2}{*}{ Ampicilina } & \multirow[t]{2}{*}{$\begin{array}{l}\text { Difusion en } \\
\text { agar con pocillo } \\
\text { y Método de } \\
\text { microdilución }\end{array}$} & \multirow[t]{2}{*}{$\begin{array}{c}\text { S. aureus } \\
\text { ATCC } 29213\end{array}$} & $\begin{array}{l}\text { M. dubia: } 25 \pm 3 \mathrm{~mm} \text { y CIM: } \\
0,08 \mathrm{mg} / \mathrm{mL}(\mathrm{n}=3) \\
\text { M. dubia: } 29 \pm 0 \mathrm{~mm} \text { y CIM: } \\
0,08 \mathrm{mg} / \mathrm{mL}(\mathrm{n}=3)\end{array}$ & \multirow[t]{2}{*}{$\begin{array}{c}\text { No } \\
\text { determinado }\end{array}$} & \multirow[t]{2}{*}{32} \\
\hline & EAPL de Sao Paulo & & & & $\begin{array}{c}\text { M. dubia con } 6 \% \text { goma arabiga a } \\
120^{\circ} \mathrm{C} \text { y } 150{ }^{\circ} \mathrm{C}: 19 \pm 1 \mathrm{~mm} \text { y CIM: } 0,16 \\
\mathrm{mg} / \mathrm{mL} \text {, respectivamente. Co: } 0,26 \mathrm{mg} / \\
\mathrm{mL} .(\mathrm{n}=3)\end{array}$ & & \\
\hline \multirow{3}{*}{$\begin{array}{l}\text { Kaneshima } \\
\text { T, et al./ } \\
\text { 2015/ Japón } \\
\text { (Artículo) }\end{array}$} & $\begin{array}{l}\text { Tres extractos y } \\
\text { un componente } \\
\text { obtenidos de la } \\
\text { cáscara (Perú) }\end{array}$ & \multirow{3}{*}{ Kanamicina } & \multirow{3}{*}{$\begin{array}{c}\text { Prueba de } \\
\text { susceptibilidad } \\
\text { por microdilución } \\
\text { en caldo }\end{array}$} & \multirow{3}{*}{$\begin{array}{l}\text { S. aureus } \\
\text { NRIC } 1135 \text {, } \\
\text { S. mutans } \\
\text { JCM } 5175 \text { y } \\
\text { C. albicans } \\
\text { JCM } 2085\end{array}$} & $\begin{array}{l}\text { Concentración inhibitoria mínima (CIM). } \\
\text { Co: } 1,56 \mu \mathrm{g} / \mathrm{mL}\end{array}$ & \multirow{3}{*}{ rminado } & \multirow{3}{*}{28} \\
\hline & Capa n-hexano & & & & $\begin{array}{l}\text { S. aureus: } 12,50 \mu \mathrm{g} / \mathrm{mL}, \text { S. mutans: } \\
25,00 \mu \mathrm{g} / \mathrm{mL} \text { y C. albicans: } \\
>100 \mu \mathrm{g} / \mathrm{mL} \\
\text { S. aureus: } 12,50 \mu \mathrm{g} / \mathrm{mL}, \text { S. mutans: No } \\
\text { probado y } \\
\text { C. albicans: }>100 \mu \mathrm{g} / \mathrm{mL}\end{array}$ & & \\
\hline & $\begin{array}{c}\text { Capa } 90 \% \\
\text { acetonitrile } \\
\text { Rhodomyrtone } \\
\text { (Componente 1) }\end{array}$ & & & & $\begin{array}{l}\text { S. aureus: } 0,78 \mu \mathrm{g} / \mathrm{mL} \text {, } \\
\text { S. mutans: } 1,56 \mu \mathrm{g} / \mathrm{mL} \text { y } \\
\text { C. albicans: }>100 \mu \mathrm{g} / \mathrm{mL}\end{array}$ & & \\
\hline
\end{tabular}

(Continuá en la página 578) 
Tabla 1. Características y resultados principales de los estudios in vitro incluidos (Viene de la página 577)

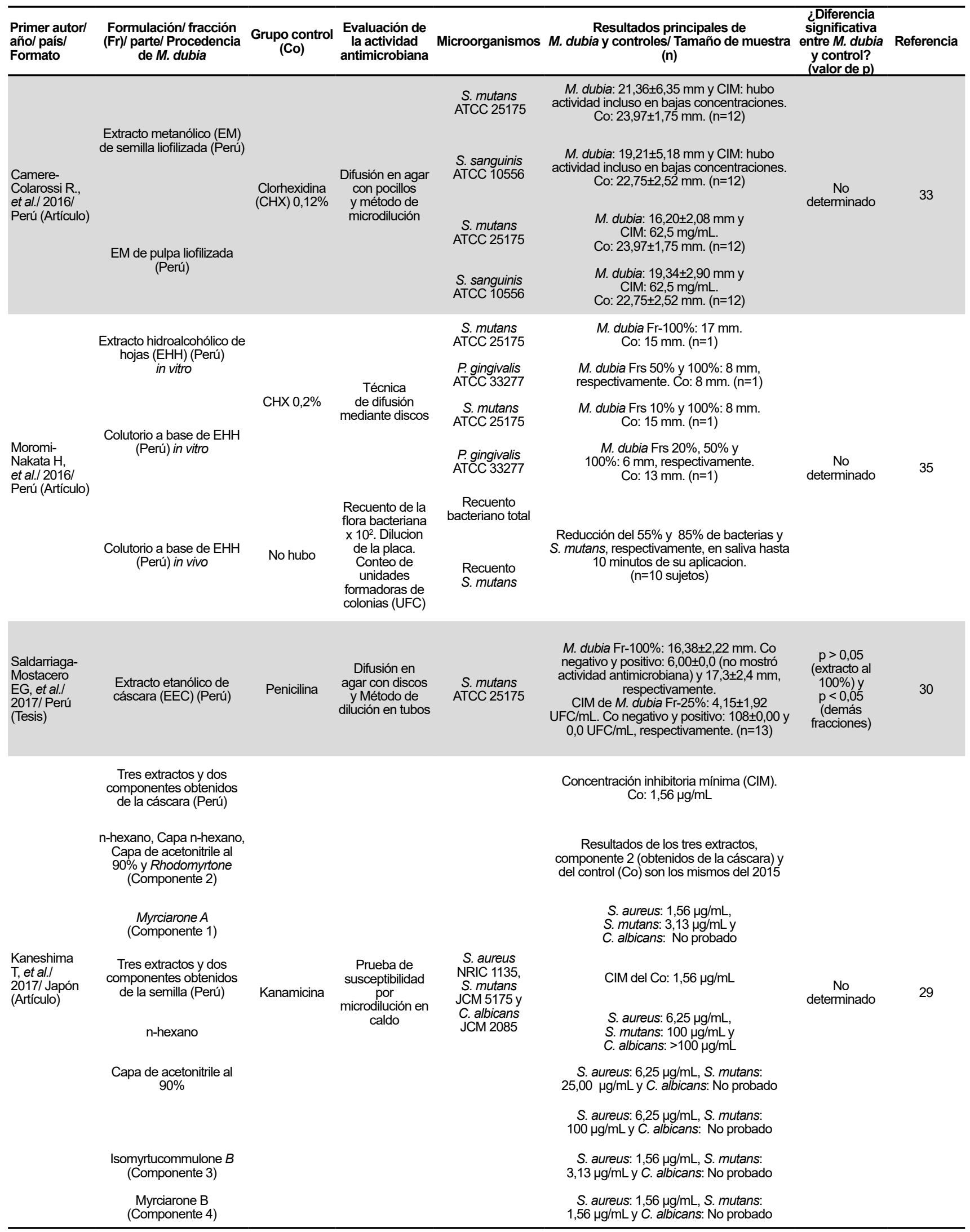


Nueve estudios usaron, predominantemente, a un antibiótico como control positivo (ampicilina ${ }^{(31,32,34,36)}$, kanamicina ${ }^{(26,28,29)}$, vancomicina $^{(27)}$ y penicilina $\left.{ }^{(30)}\right)$, uno de ellos incluyó al fluconazol ${ }^{(27)}$ y otro un alcohol (solo indicó que era de $96^{\circ}$ ) ${ }^{(35)}$, encontrando que la actividad de $M$. dubia fue en su mayoría similar ${ }^{(29,30)}$ o superior ${ }^{(28,29,31,33,36)}$ a dichos antibióticos, dos estudios usaron la clorhexidina ( $\mathrm{CHX}$ ) (en solución), como control positivo ${ }^{(33,35)}$, y un estudio utilizó un control negativo. Finalmente, el análisis estadístico de dicho estudio, no encontró diferencia significativa entre la actividad de cuatro fracciones de un extracto de cáscara de $M$. dubia versus penicilina ${ }^{(30)}$.

El sesgo de los estudios fue evaluado mediante el criterio descrito por Joana Briggs Institute ${ }^{(25)}$, utilizado en revisiones sistemáticas de estudios in vitro ${ }^{(37-39)}$. La Figura 2 muestra el riesgo de sesgo encontrado en los estudios incluidos en esta revisión. Al analizarlos se observó que todos los estudios presentaron un «no claro» riesgo de sesgo para la asignación aleatoria, ocultamiento de la lista de asignación aleatoria y cegamiento de los evaluadores. Respecto, a un análisis estadístico apropiado, solo fue ejecutado en uno de

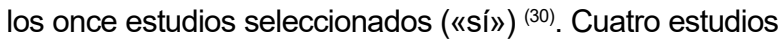
no requirieron un análisis estadístico porque generaron resultados estimados puntualmente ${ }^{(26,28,29,35)}$. Además, en cinco estudios, el análisis estadístico fue "no claro" (27,31-33,36) y uno no utilizó el análisis estadístico apropiado ${ }^{(34)}$ («no»). Las demás directrices, que incluyen la comparación basal entre grupos experimentales, tratamientos y mediciones del evento idénticos fueron ejecutados en forma confiable y comparable, mostrando un «si» bajo riesgo de sesgo en todos los estudios.

En uno de los estudios, se observó que pese a la pérdida de fitoquímicos ocurrida en la pulpa de M. dubia, debido a que fue sometida a procesos de deshidratación, el polvo de la pulpa aún mantuvo altos niveles de fenólicos, ácido ascórbico, proantocianidinas y actividad antimicrobiana (31). Asimismo, un artículo concluyó que extractos de semilla y cáscara inhibieron a $S$. aureus, siendo responsables los constituyentes lipofílicos ${ }^{(26)}$. Otras dos publicaciones demostraron la actividad antimicrobiana de compuestos fenólicos de $M$. dubia; en la primera, identificaron dichos compuestos en la pulpa liofilizada y hallaron que tenían un coeficiente de correlación alto con la inhibición de $S$. aureus $\left(r^{2}=0,906-0,971\right)^{(32)}$. Y en la segunda, el polvo de semilla+cáscara de $M$. dubia presentaron compuestos fenólicos y polifenoles, obtenidas por liofilización y aire caliente, respectivamente, indicando que podrían desempeñar una función al inhibir $S$. aureus ${ }^{(36)}$. Finalmente, dos estudios aislaron cuatro acilfloroglucinoles, fitoquímicos antimicrobianos, en la cáscara ${ }^{(28,29)}$ y semilla ${ }^{(29)}$ de M. dubia, respectivamente, los cuales evidenciaron fuertes actividades antimicrobianas contra cinco bacterias grampositivas entre ellas $S$. mutans y $S$. aureus ${ }^{(28,29)}$.

\section{DISCUSIÓN}

La presente revisión sistemática demostró, mayoritariamente, que la $M$. dubia presenta actividad antimicrobiana contra microorganismos, grampositivos $(\mathrm{G}+)^{(26-36)}$ y sólo un gramnegativo (G-) ${ }^{(35)}$ y un hongo ${ }^{(27)}$ de la cavidad oral. Así también, contra microrganismos G- no pertenecientes a la cavidad oral ${ }^{(28,29,40)}$, lo que nos sugiere que la $M$. dubia podría inhibir patógenos G- relevantes en el desarrollo de la EP. Por lo tanto, es necesario realizar más estudios, in vitro, sobre otras bacterias G+ y G- relacionada a CD y EP, respectivamente; aplicando otras metodologías/técnicas y cuyo grupo control sea la clorhexidina. Luego, debería ejecutarse estudios en un ambiente in vivo, como la boca. Particularmente, se podría estudiar su sustantividad, que es la propiedad que permite que persista su acción terapéutica el mayor tiempo posible en la cavidad oral. Por ende, más estudios in vitro, in vivo y posteriormente clínicos deberían realizarse en vista de su potencial actividad antimicrobiana.

La M. dubia ha sido utilizada, tradicionalmente, por comunidades indígenas de Loreto (Perú) para curar gingivitis ${ }^{(41)}$ y porque mantiene la salud de las encías ${ }^{(42)}$. Actualmente, es considerada una super fruta, debido a que posee diversos fitoquímicos y porque mejora la calidad de vida de las personas. En los últimos años se han estudiado extractos del fruto completo y sus partes, demostrando sus efectos contra diferentes patologías, incluyendo su acción antimicrobiana contra patógenos orales ${ }^{(43)}$. Los estudios realizados en microorganismos orales sugieren la actividad antimicrobiana de M. dubia ${ }^{(26-36)}$.

Se estudió a las bacterias anaerobias estrictas, por ser consideradas los principales agentes etiológicos de la EP ${ }^{(44)}$. Además, de bacterias $\mathrm{G}+$ por ser agentes causales de la CD ${ }^{45}$ y otros componentes microbianos que conforman la PD. Particularmente, se decidió evaluar la actividad antimicrobiana porque es específica en los microorganismos de la cavidad oral, por ejemplo, las comunidades polimicrobianas representativas de la $C D$ y periodontitis muestran una sofisticada integración estructural y funcional, confiriendo un estado casi orgánico a estos microorganismos ${ }^{(46)}$. No se estudió otras propiedades, como la actividad antiinflamatoria debido a que no es especifica en la cavidad oral y puede evaluarse mediante otros modelos experimentales, aplicados frecuentemente al organismo completo.

En relación al análisis de sesgo de los estudios incluidos, existe una percepción de sesgo en la mayoría de ellos, porque no se cumplió con las directrices de asignación aleatoria, ocultamiento de la lista de asignación aleatoria y cegamiento de los evaluadores. Sin embargo, dichas directrices, normalmente no se cumplen en las investigaciones microbiológicas in vitro. Respecto a las demás directrices, si fueron aplicadas; excepto el de análisis estadístico, que no se cumplió en todos los estudios, porque no compararon los grupos experimentales versus controles positivos ${ }^{(26-29,31-36)}, y$ 
en otros no requería ejecutarlo porque el método empleado para medir el evento principal generó resultados estimados puntualmente (presentaron un único valor) ${ }^{(26,28,29,35)}$. Sin embargo, los resultados nos mostrarían el potencial para generar evidencia.

Aún se desconoce el mecanismo de acción de los fitoquímicos de $M$. dubia, identificados en esta revisión y habitualmente estudiados como nuevos constituyentes antimicrobianos ${ }^{(28,29,47)}$. No obstante, se ha evidenciado que $\mathrm{G}+$, como $S$. aureus, mostraron sensibilidad microbiana frente a compuestos fenólicos de frutas, los cuales actúan mediante varios mecanismos incluyendo desestabilización de la membrana celular e inhibición de encimas clave ${ }^{(47)}$.

Una serie de compuestos fenólicos fueron la mejor elección, como suplemento, durante la terapia antibiótica, porque aceleraron la actividad antimicrobiana e inhibieron la producción excesiva de especies reactivas de oxígeno por agentes antimicrobianos ${ }^{(48)}$. Además, estudios en animales indicaron que muchos de ellos mejoraron o previnieron el desarrollo de EP en términos de inflamación y destrucción periodontal ${ }^{(49)}$. Los compuestos lipofílicos serían relevantes en el potencial de dos extractos para prevenir la erosión dental ${ }^{(50)}$. A menudo el término polifenoles es usado como sinónimo de compuestos fenólicos, pero sólo se refiere a las moléculas que soportan al menos dos anillos fenólicos ${ }^{(51)}$. Estudios in vitro ${ }^{(52)}$, de biología celular, en animales y humanos han demostrado que polifenoles dietéticos selectos tienen importantes propiedades antimicrobianas, antioxidantes y antiinflamatorias que mejoran los marcadores clínicos de la periodontitis ${ }^{(53)}$. Por último, los acilfloroglucinoles derivados de diversas plantas medicinales exhibieron actividades antimicrobianas y antifúngicas ${ }^{(54)}$ y poseen características estructurales importantes que le confieren actividades antimicrobianas contra cepas resistentes de $S$. aureus ${ }^{(55)}$.

En la presente revisión se encontraron ciertas limitaciones, debido a la heterogeneidad en la intervención y en los resultados. En la primera, se refiere a diferencias en la $M$. dubia, respecto a su lugar de procedencia, tipo de extracto, partes del fruto utilizadas y diferentes controles positivos. En el segundo, diferencias en la metodología para cuantificar la actividad antimicrobiana y en sus unidades de medida. Además, como los resultados se basaron en estudios experimentales in vitro, no conducen a la aplicación clínica directa, pero si constituye un paso previo necesario. No obstante, a las limitaciones en la intervención y resultados, la actividad antimicrobiana de $M$. dubia generalmente evidenció ser similar ${ }^{(29,30)}$ y/o superior $(28,29,31,32,36)$ a antibióticos, sugiriendo así su potencial antimicrobiano en la cavidad oral. A pesar de que en los dos únicos estudios en donde se le comparó con $\mathrm{CHX}{ }^{(33,35)}$, no mostró superioridad. Esto indica la necesidad de estudiarlo más en el ámbito odontológico y así poder medir su actividad antimicrobiana versus antisépticos orales convencionales. Cabe mencionar que existen revisiones de literatura de $M$. dubia ${ }^{(56,23,57)}$, pero esta es la primera revisión sistemática que evalúa su actividad antimicrobiana contra microorganismos de la cavidad oral.

Se puede concluir que $M$. dubia tiene potencial como antimicrobiano en la cavidad oral para controlar caries dental y enfermedad periodontal, además, de reducir el recuento total de microorganismos. Para corroborar su efecto antimicrobiano, se debería estudiarla en otras formulaciones como antisépticos (soluciones), dentífricos o en goma de mascar, etc. El pobre diseño metodológico de los estudios incluidos en la presente revisión sistemática, no posibilita su uso como antimicrobiano directamente en la cavidad oral. Recomendamos ejecutar más estudios con mejor diseño y bajo riesgo de sesgo.

Contribuciones de los autores: KPA participó en la concepción y diseño del artículo, recolección de datos, redacción y revisión crítica del artículo, análisis e interpretación de los resultados y en la aprobación de la versión final. MPV participó recolección de datos, en la revisión crítica del artículo y en la aprobación de la versión final. AG participó en la recolección de datos, redacción del artículo, revisión crítica del artículo y en la aprobación de la versión final. JMUT participó en la recolección de datos, en la revisión crítica del artículo y en la aprobación de la versión final.

Fuentes de financiamiento: Autofinanciado.

Conflictos de interés: los autores declaran no tener conflictos de interés en relación a los contenidos de este documento

Material suplementario: Disponible en la versión electrónica de la RPMESP.

\section{REFERENCIAS BIBLIOGRÁFICAS}

1. Doherty R. Biofilms: What does subgingival plaque look like? Br Dent J. 2016 Jul;221(1):16. doi: https://doi. org/10.1038/sj.bdj.2016.487.

2. Garcia SS, Blackledge MS, Michalek S, Su L, Ptacek T, Eipers P. Targeting of Streptococcus mutans biofilms by a novel small molecule prevents dental caries and preserves the oral microbiome. J Dent Res. 2017;96(7):807-14. doi: https:// doi.org/10.1177/0022034517698096.

3. Hajishengallis G. Periodontitis: from microbial immune subversion to systemic inflammation. Nat Rev Immunol. 2015;15(1):30-44. doi: https://doi.org/10.1038/nri3785.
4. Wilder RS, Bray KS. Improving periodontal outcomes: merging clinical and behavioral science. Periodontol 2000. 2016;71:65-81. doi: https://doi. org/10.1111/prd.12125.

5. Mouchrek Junior JC, Nunes LH, Arruda CS, Rizzi Cde C, Mouchrek $\mathrm{AQ}$, Tavarez RR, et al. Effectiveness 
of oral antiseptics on tooth biofilm: a study in vivo. J Contemp Dent Pract. 2015 Aug;16(8):674-8. doi: https://doi. org/10.5005/jp-journals-10024-1739.

6. Wessel SW, van der Mei HC, Maitra A, Dodds MW, Busscher HJ. Potential benefits of chewing gum for the delivery of oral therapeutics and its possible role in oral healthcare. Expert Opin Drug Deliv. 2016;3:1-11. doi: https://doi.org/10.108 0/17425247.2016.1193154.

7. Supranoto SC, Slot DE, Addy M, Van der Weijden GA. The effect of chlorhexidine dentifrice or gel versus chlorhexidine mouthwash on plaque, gingivitis, bleeding and tooth discoloration. Int J Dent Hyg. 2015;13(2):83-92. doi: https://doi. org/10.1111/idh.12078.

8. Tartaglia GM, Kumar S, Fornari CD, Corti E, Connelly ST. Mouthwashes in the 21st century: a narrative review about active molecules and effectiveness on the periodontal outcomes. Expert Opin Drug Deliv. 2017;14(8):973-82. doi: https://doi. org/10.1080/17425247.2017.1260118.

9. Kulik EM, Waltimo T, Weiger R, Schweizer I, Lenkeit K, Filipuzzi-Jenny E, et al. Development of resistance of mutans streptococci and Porphyromonas gingivalis to chlorhexidine digluconate and amine fluoride/stannous fluoride-containing mouthrinses, in vitro. Clin Oral Investig. 2015;19(6):1547-53. doi: https://doi. org/10.1007/s00784-014-1379-y.

10. CastroJC,MaddoxJD, Cobos M, ImánSA. Myrciaria dubia "camu camu" fruit: healthpromoting phytochemicals and functional genomic characteristics. Breeding and Health Benefits of Fruit and Nut Crops; London: IntechOpen; 2018. doi: https:// doi.org/10.5772/intechopen.73213.

11. Kaneshima T, Myoda T, Toeda K, Fujimori T, Nishizawa M. Antioxidative constituents in camu-camu fruit juice residue. Food Sci Technol Res. 2013;19(2):223-8. doi: https://doi.org/10.3136/fstr.19.223.

12. Kaneshima T, Myoda T, Nakata M, Fujimori T, Toeda K, Nishizawa M. Antioxidant activity of C-Glycosidic ellagitannins from the seeds and peel of camu-camu (Myrciaria dubia). LWT-Food Sci Technol. 2016;69:7681. doi: https://doi.org/10.1016/j. lwt.2016.01.024.

13. Cunha-Santos ECE, Viganó J, AndradeNeves D, Martínez J, Teixeira-Godoy H. Vitamin C in camu-camu [Myrciaria dubia (H.B.K.) McVaugh]: evaluation of extraction and analytical methods. Food Res Int. 2019;115:160-6. doi: https://doi. org/10.1016/j.foodres.2018.08.031.

14. Fidelis M, Santos JS, Escher GB, Vieira do Carmo M, Azevedo L, Cristina da
Silva M, et al: In vitro antioxidant and antihypertensive compounds from camu-camu (Myrciaria dubia McVaugh, Myrtaceae) seed coat: A multivariate structure-activity study. Food Chem Toxicol. 2018;120:479-90. doi: https:// doi.org/10.1016/j.fct.2018.07.043.

15. Yazawa K, Suga K, Honma A, Shirosaki M, Koyama T. Anti-inflammatory effects of seeds of the tropical fruit camu-camu (Myrciaria dubia). J Nutr Sci Vitaminol (Tokyo). 2011;57(1):104-7. doi: https:// doi.org/10.3177/jnsv.57.104.

16. Inoue $T$, Komoda $H$, Uchida $T$, Node K. Tropical fruit camu-camu (Myrciaria dubia) has anti-oxidative and antiinflammatory properties. J Cardiol. 2008;52(2):127-32. doi: https://doi. org/10.1016/j.jjcc.2008.06.004.

17. Fujita A, Sarkar D, Genovese MI, Shetty K. Improving anti-hyperglycemic and anti-hypertensive properties of camucamu (Myriciaria dubia Mc. Vaugh) using lactic acid bacterial fermentation. Process Biochem. 2017;59(B):13340. doi: https://doi.org/10.1016/j. procbio.2017.05.017.

18. Anhê FF, Nachbar RT, Varin TV, trottier J, Dudonné S, le Barz M, et al. Treatment with camu-camu (Myrciaria dubia) prevents obesity by altering the gut microbiota and increasing energy expenditure in diet-induced obese mice. Gut. 2019;68(3):453-64. doi: http:// dx.doi.org/10.1136/gutjnl-2017-315565.

19. Nascimento OV, Boleti APA, Schwertz M, Lima ES. Dietary supplementation with camu-camu and continuous exercises in the treatment of obesity. Rev Nutr. 2018;31(1):25-33. doi: https://doi. org/10.1590/1678-98652018000100003.

20. Asmat-Aguirre SN, Benites-Carranza CY. Efecto del fruto de Myrciaria dubia (H.B.K) Mc Vaugh sobre cáncer colorrectal inducido en Rattus Norvegicus Var. Albinus [Tesis]. Trujillo, Perú: Universidad Nacional de Trujillo, Facultad de Farmacia y Bioquímica; 2017. Disponible en: http://dsapce.unitru.edu. pe/handle/UNITRU/7437.

21. Macedo-Ríos R, Mendoza-Acuña JB. Actividad inmunoestimulante del fruto de Myrciaria dubia H.B.K Mc Vaugh "Camu camu", en ratas albinas Holtzmann, Iquitos 2015 [Tesis]. Iquitos, Perú: Universidad Nacional de la Amazonía Peruana, Facultad de Farmacia y Bioquímica; 2015. Disponible en: http:// repositorio.unapiquitos.edu.pe/handle/ UNAP/3868.

22. Chapple IL, Bouchard P, Cagetti MG, Campus G, Carra MC, Cocco F. Interaction of lifestyle, behavior or systemic diseases with dental caries and periodontal diseases: consensus report of group 2 of the joint EFP/ORCA workshop on the boundaries between caries and periodontal diseases. J Clin Periodontol. 2017;44(Suppl. 18):S39-S51. doi: https:// doi.org/10.1111/jcpe.12685.

23. Jafer M, Patil S, Hosmani J, Bhandi SH, Chalisserry EP, Anil S. Chemical plaque control strategies in the prevention of biofilmassociated oral diseases. J Contemp Dent Pract. 2016;17(4):337-43. doi: https://doi. org/10.5005/jp-journals-10024-1851.

24. Liberati A, Altman DG, Tetzlaff J, Mulrow C, Gøtzsche PC, Ioannidis JPA, et al. The PRISMA statement for reporting systematic reviews and metaanalyses of studies that evaluate health care interventions: Explanation and Elaboration. J Clin Epidemiol [Internet]. 2009 Oct [cited 2019 Set 01];62(10):e1e34. Available from: https://journals.plos. org/plosmedicine/article ?id=10.1371/ journal.pmed.1000100. doi: https://doi. org/10.1371/journal.pmed.1000100.

25. The Joanna Briggs Institute. Joanna Briggs Institute reviewer's manual 2014 [Internet]. South Australia: The Joanna Briggs Institute; 2014 [cited 2019 Ago 18]. Available from: https://nursing. lsuhsc.edu/JBI/docs/ReviewersManuals/ Economic.pdf.

26. Myoda T,FujimuraS,ParkB,NagashimaT, Nakagawa J, Nishizawa M. Antioxidative and antimicrobial potential of residues of camu-camu juice production. J Food Agri Environ. 2010;8(2):304-7.

27. Castillo-Carranza CN, Mejía-Delgado EM. Efecto inhibitorio in vitro de Myrciaria dubia "camu-camu" sobre Staphylococcus aureus y Candida albicans [Tesis Bachiller en medicina]. Trujillo: Universidad Nacional de Trujillo; 2013. Disponible en: http://dspace.unitru.edu. pe/handle/UNITRU/232.

28. Kaneshima T,Myoda T,NakataM,Fujimori T, Toeda K, Nishizawa M. Rhodomyrtone, an antimicrobial acylphloroglucinol, in the peel of Myrciaria dubia (Camu-camu). Food Preserv Sci. 2015;41(2):71-6.

29. Kaneshima T, Myoda T, Toeda K, Fujimori T, Nishizawa M. Antimicrobial constituents of peel and seeds of camu-camu (Myrciaria dubia). Biosci Biotechnol Biochem. 2017;81(8):1461-5. doi: https://doi.org/10 $.1080 / 09168451.2017 .1320517$.

30. Saldarriaga-Mostacero EG. Efecto antibacteriano in vitro del extracto etanólico de Myrciaria dubia (camu camu) sobre Streptococcus mutans (ATCC 25175) [Tesis]. Trujillo, Perú: Universidad Nacional de Trujillo, Facultad de Odontología; 2017. Disponible en: http://dspace.unitru.edu. pe/handle/UNITRU/7996 
31. Fujita A, Borges K, Correia R, Gombossy de Melo Franco BD, Genovese MI. Impact of spouted bed drying on bioactive compounds, antimicrobial and antioxidant activities of commercial frozen pulp of camucamu (Myrciaria dubia Mc. Vaugh). Food Res Int. 2013;54:495-500. doi: https://doi. org/10.1016/j.foodres.2013.07.025.

32. Fujita A, SarkarD, Wu S, Kennelly E, Shetty $\mathrm{K}$, Genovese MI. Evaluation of phenoliclinked bioactives of camu-camu (Myrciaria dubia Mc. Vaugh) for antihyperglycemia, antihypertension, antimicrobial properties and cellular rejuvenation. Food Res Int. 2015 Nov;77(2):194-203. doi: https:// doi.org/10.1016/j.foodres.2015.07.009.

33. Camere-Colarossi R, Ulloa-Urizar G, Medina-Flores D, Caballero-García S, Mayta-Tovalino F, del Valle-Mendoza J. Antibacterial activity of Myrciaria dubia (Camu camu) against Streptococcus mutans and Streptococcus sanguinis. Asian Pac J Trop Biomed [Internet]. 2016 Sep [cited 2018 Sep 19];6(9):[about 1 p.]. doi: https://doi. org/10.1016/j.apjtb.2016.07.008.

34. Mori TJ, Ruiz E, Bardales J, Garcia M, Tresierra A, Arévalo L, col. Efecto antimicrobiano de Myrciaria dubia "camucamu" y Cyperus luzula "piripiri" sobre microorganismos patógenos. Conoc Amaz. 2013;4(1):49-57.

35. Moromi-Nakata H, Perfecto DR, Cadillo EM, Alvarado EC, Espinoza F. Efectividad in vitro e in vivo de un colutorio a base de Myrciaria dubia "camu camu" sobre bacterias de importancia oral. Theorema (Lima, Segunda época, En línea) [Internet]. 2016 [citado 19 de setiembre de 2018];(1):[1 página]. Disponible en: http://revistasinvestigacion.unmsm.edu. pe/index.php/Theo/article/view/11941

36. De Azevêdo, JCS, Fujita A, de Oliveira EL, Genovese MI, Correia RTP. Dried camu-camu (Myrciaria dubia HBK McVaugh) industrial residue: A bioactiverich Amazonian powder with functional attributes. Food Res Int. 2014;62:93440. doi: https://doi.org/10.1016/j. foodres.2014.05.018.

37. Shringeri PI, Fareed N, Battur H, Khanagar S. Role of probiotics in the treatment and prevention of oral malodor/halitosis: A systematic review. J Indian Assoc Public Health Dent. 2019;17(2):92-6. doi: https://doi.org/10.4103/jiaphd. jiaphd_171_18.

38. Dadashi M, Nasiri MJ, Fallah F, Owlia P, Hajikhani B, Emaneini M, et al. Methicillinresistant Staphylococcus aureus (MRSA) in Iran: a systematic review and meta-analysis. J Glob Antimicrob Resist. 2018;12:96103. doi: https://doi.org/10.1016/j. jgar.2017.09.006.
39. Dludla PV, Nkambule BB, Dias SC, Johnson R. Cardioprotective potential of $\mathrm{N}$-acetyl cysteine against hyperglycaemiainduced oxidative damage: a protocol for a systematic review. Syst Rev. 2017;6:96. doi: 10.1186/s13643-017-0493-8.

40. López-Mata AE. Efecto antibacteriano del zumo de Myrciaria dubia, Citrus grandes y Citrus reticula sobre Escherichia coli y Salmonella tiphy. CIENTIFI-K. 2017;5(1):87-92. doi: dx.doi. org/10.18050/Cientifi-k.v5n1a10.2017.

41. Flores D. Uso histórico: Camu camu, Myrciaria dubia (H.B.K) Mc Vaugh [Internet] 2010. Disponible en: http:// repositorio.promperu.gob.pe/repositorio/ bitstream/handle/123456789/1367/ U s o h is torico c a m u camu_2010_keyword_principal. pdf ? sequence $=1$ \& is Allowed $=\mathrm{y}$.

42. Pinedo M, Delgado C, Farroñay R, Del Castillo D, Iman $S$, col. Camu-camu (Myrciaria dubia, Myrtaceae). Aportes para su aprovechamiento sostenible en la Amazonia Peruana. Lima: Instituto de Investigaciones de la Amazonia Peruana; 2011. Disponible en: http://repositorio. iiap.org.pe/bitstream/IIAP/120/2/ Pinedo_Libro_2011.pdf.

43. Arellano-Acuña E, Rojas-Zavaleta I, PaucarMenacho LM. Camu-camu (Myrciaria dubia): Fruta tropical de excelentes propiedades funcionales que ayudan a mejorar la calidad de vida. Sci Agropecu. 2016;7(4):433-43. doi: http://dx.doi. org/10.17268/sci.agropecu.2016.04.08.

44. Hurtado-Camarena A, Bojórquez-Anaya Y, Montaño-Pérez ML, López-Mendoza JA. Bacterias asociadas a enfermedades periodontales. Oral. 2016;17(54):1374-8.

45. Wang W, Tao R, Tong Z, Ding Y, Kuang R, Zhai S, et al. Effect of a novel antimicrobial peptide chrysophsin-1 on oral pathogens and Streptococcus mutans biofilms. Peptides. 2012;33:212-9. doi: https://doi. org/10.1016/j.peptides.2012.01.006.

46. Lamont RJ, Koo H, Hajishengallis G. The oral microbiota: dynamic communities and host interactions. Nat Rev Microbiol. 2018;16(12):745-59. doi: https://doi. org/10.1038/s41579-018-0089-x.

47. Caillet $S$, Côté J, Sylvain JF, Lacroix M. Antimicrobial effects of fractions from cranberry products on the growth of seven pathogenic bacteria. Food Control. 2012 Feb;23(2):419-28. doi: 10.1016/j. foodcont.2011.08.010.

48. Mandal SM, Dias RO, Franco OL. Phenolic compounds in antimicrobial therapy.J Med Food. 2017;20(10):1031-8. doi: https:// doi.org/10.1089/jmf.2017.0017.
49. Varela-López A, Bullón P, Giampieri F, Quiles JL. Non-nutrient, naturally occurring phenolic compounds with antioxidant activity for the prevention and treatment of periodontal diseases. Antioxidants. 2015;4(3):447-81. doi: 10.3390/antiox4030447.

50. Weber MT, Hannig M, Pötschke S, Höhne F, Hannig C. Application of plant extracts for the prevention of dental erosion: An in situ/in vitro Study. Caries Res. 2015;49(5):477-87. doi: https://doi. org/10.1159/000431294.

51. Quideau S, Deffieux D, Douat-Casassus C, Pouysengu L. Plant polyphenols: chemical properties, biological activities, and synthesis. Angew Chem Int Ed Engl. 2011 Jan;50(3):586-621. doi: https://doi. org/10.1002/anie.201000044.

52. Daglia M. Polyphenols as antimicrobial agents. Curr Opin Biotechnol. 2012;23(2):174-81. doi: https://doi. org/10.1016/j.copbio.2011.08.007.

53. Basu A, Masek E, Ebersole JL. Dietary polyphenols and Periodontitis-A Mini-Review of Literature. Molecules. 2018 Jul;23(7):1786. doi: https://doi. org/10.3390/molecules23071786.

54. Pal Singh I, Bharate SB. Phloroglucinol compounds of natural origin. Nat Prod Rep. 2006;23(4):558-91. doi: 10.1039/ b600518g.

55. Rahman MM, Shiu WKP, Gibbons $S$, Malkinson JP. Total synthesis of acylphloroglucinols and their antibacterial activities against clinical isolates of multidrug resistant (MDR) and methicillinresistant strains of Staphylococcus aureus. Eur J Med Chem. 2018 Jul;155:25562. doi: https://doi.org/10.1016/j. ejmech.2018.05.038.

56. Akter MS, Oh S, Eun JB, Ahmed M. Nutritional compositions and health promoting phytochemicals of camu-camu (Myrciaria dubia) fruit: A review. Food Res Int. 2011;44(7):1728-32. doi: https:// doi.org/10.1016/j.foodres.2011.03.045.

57. Langley PC, Pergolizzi JV Jr, Taylor R Jr, Ridgway C. Antioxidant and associated capacities of camu camu (Myrciaria dubia): a systematic review. J Altern Complement Med. 2015;21(1):8-14. doi: https://doi. org/10.1089/acm.2014.0130.

Correspondencia: Pardo-Aldave Karina Dirección. Av. Los Halcones 520. Bellavista, Callao. Perú.

Teléfono: 01-14510225, 9973-10819

Correo:khiezay@yahoo.es,infokarina@gmail.com 